\title{
A Two-way Route Repair Scheme Based on Hop-count
}

\author{
Xuewen Wu, Xiaokai Zhu, Fei Kong \\ College of Computer and Information, Hohai University, Nanjing, 211100, China \\ e-mail: hhuzxk@126.com
}

\begin{abstract}
Considering the limitations of one-way route repair in Mobile Ad Hoc Networks, this paper proposes a novel two-way route repair scheme based on hop-count. This scheme takes the hop differences between the backward and forward node near the broken link as judging rules which decide the direction to repair and thereby shortens the distances of routing maintenance effectively. The simulation results show that the scheme has a better performance in end-to-end packet delay and control overhead than the previous schemes.
\end{abstract}

Keywords-mobile Ad-hoc networks; hops differences; two-way; route repair; routing maintenance

\section{INTRODUCTION}

Mobile Ad-hoc Networks (MANETs) is a multi-hop, self-organizing and wireless network with dynamic changing of topology. One of the key challenges in this network is routing protocol in which a lot of one-way protocols exist such as Ad-hoc On Demand Distance Vector (AODV) protocol [1-3] and Dynamic Source Routing (DSR) protocol [4-7]. They are both classic oneway on-demand routing protocols.

There are three main route repair mechanisms for oneway routing protocols including Source Repair, Local Repair and Backup Routing Repair. An improvement of DSR protocol based on time to live (TTL) in [8] was studied, but it would lead to high delay problem. In [9] the authors proposed an enhanced BCOAODV protocol and considered the backup paths and link loads as the performance metrics to balance the network traffic effectively. However it cost more to maintain multiple backup paths. In [10], a local repair called PATCH was proposed which used less overhead to achieve rapid repair of broken link but could only be used for source routing protocols.

The main differences in these three mechanisms are that when links break, the starting nodes of route discovery and search conditions are different. Meanwhile, all of them adopt one-way repair mode of route. No matter where the broken place is, route repair is inflexible from the upstream to the downstream. So it will have a higher overhead and delay when the broken place is far from the destination node.

In our work, we explore the hop-count difference of backward and forward nodes and put forward repair direction judging criterions to achieve the goal of making the nodes near the broken link autonomously, dynamically repair the route upward or downward.
This following paper is organized as follows. Section II details our scheme. Section III shows examples to illustrate our scheme. Section IV presents simulation and related analysis. Finally, we conclude in Section V.

\section{OUR PROPOSED SCHEME}

\section{A. Route Repair Judging Rules}

Here we define the destination node and source node as $\mathrm{D}$ and $\mathrm{S}$. The nodes upstream and downstream of the link break are defined as N1 and N2 respectively.

Besides, $\operatorname{Hop}(X, D)$ and $\operatorname{Hop}(X, S)$ are defined as the hop distances between any node $X$ along the route with $\mathrm{D}$ or $\mathrm{S}$. The difference of hop-count

$$
\Delta H(X)=H o p(X, S)-H o p(X, D)
$$

Obviously we can get two facts.

- $\operatorname{Hop}(S, S)=0 \operatorname{Hop}(D, D)=0$;

- $\Delta H(N 2)=\Delta H(N 1)+2$, because

$$
\begin{aligned}
\Delta H(N 2) & =\operatorname{Hop}(N 2, S)-\operatorname{Hop}(N 2, D) \\
& =(\operatorname{Hop}(N 1, S)+1)-(\operatorname{Hop}(N 1, D)-1) \\
& =\Delta H(N 1)+2
\end{aligned}
$$

repair from the broken place to the nodes $\mathrm{D}$ and $\mathrm{S}$ are called "Route Repair Downstream" (denoted by strategy SD) and "Route Repair Upstream" (denoted by strategy SU).

The distances from the place where the link break occurred to nodes D or S are defined as lengths L1 and L2.

- If $\mathrm{L} 1=\mathrm{L} 2$, then $\Delta H(N 1)=-1, \Delta H(N 2)=1$. So node N1 should use strategy SD.

- If $\mathrm{L} 1<\mathrm{L} 2, \Delta H(N 2)>0$, then $\Delta H(N 2)>1$,

$\Delta H(N 1)>-1$ and $\mathrm{N} 1$ should use strategy $\mathrm{SD}$.

- If $\mathrm{L} 1>\mathrm{L} 2, \Delta H(N 1)<0$, then $\Delta H(N 1)<-1$,

$\Delta H(N 2)<1$ and $\mathrm{N} 2$ should use strategy SU.

Therefore, the two judging rules are as follows.

a) If $\Delta H(N 1) \geq-1$ and $\Delta H(N 2) \geq 1$, strategy $\mathrm{SD}$ will be used ;

b) If $\Delta H(N 1)<-1$ and $\Delta H(N 2)<1$, strategy SU will be used. 


\section{B. Route Repair Process}

Route discovery means the establishing of new routes and route repair means the maintenance of the route.

In the case of a broken link, nodes $\mathrm{N} 1$ and $\mathrm{N} 2$ choose the strategy SD or strategy SU based on the judging rules. Fig .1 shows the flow chart of the key points of the whole scheme.

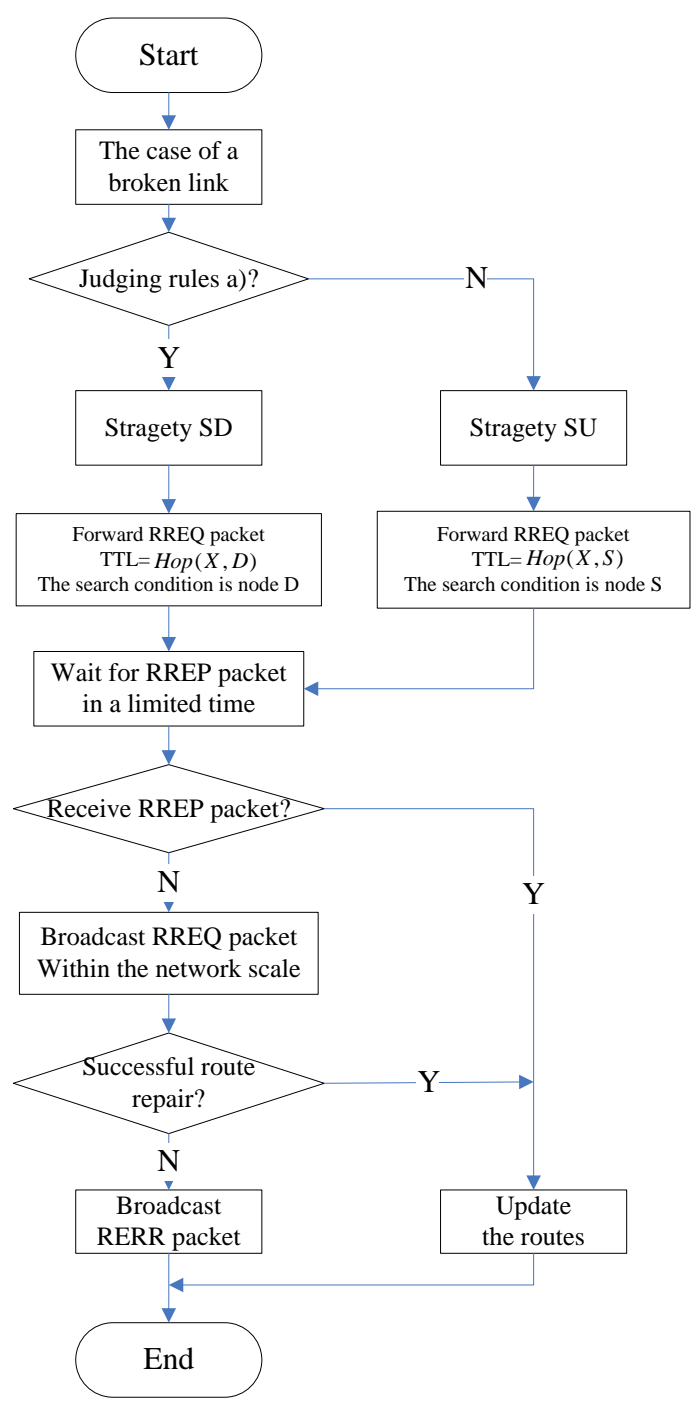

Figure 1. The scheme of routing maintenance

\section{EXAMPLES FOR OUR SCHEME}

In routing protocols, there are three frequently used control messages including Route Request (RREQ), Route Replay (RREP) and Routing Error (RERR). During the establishing of new route, the hop-count in RREQ and RREP packet is defined as HC.

\section{A. Strategy $S D$}

Fig .2 shows the process of downward route repair. In this scenario, the route links are as follows.

$$
S \leftrightarrow A \leftrightarrow B \leftrightarrow C \leftrightarrow E \leftrightarrow D
$$

A link break occurs between nodes $\mathrm{C}$ and $\mathrm{E}$.
Considering $\Delta H(C)=3-2=1>-1$, node $\mathrm{C}$ should choose the $\mathrm{SD}$ approach according to judging rules a).Within the scale of $T T L=H o p(C, D)=2$, node $\mathrm{C}$ broadcasts RREQ message to search for the destination D. The source IP address is set to the source node $\mathrm{S}$ and HC's initial value is set to 4. It's necessary for node $\mathrm{J}$ to update or add the reverse route to node $S$ because it has no path to node $\mathrm{D}$. Then node $\mathrm{J}$ should continue forwarding RREQ packet to node $\mathrm{K}$ with $\mathrm{TTL}=1, \mathrm{HC}=5$. When node $\mathrm{K}$ has received corresponding RREQ packet and node $\mathrm{J}$ discovers that it has a new route path to node $\mathrm{D}$, node $\mathrm{J}$ is supposed to send RREP packet along the new path in the opposite direction. After node $\mathrm{C}$ having got the RREP packet from node $J$ and the routes having updated, the new end-to-end route is

$$
S \leftrightarrow A \leftrightarrow B \leftrightarrow C \leftrightarrow J \leftrightarrow K \leftrightarrow D
$$

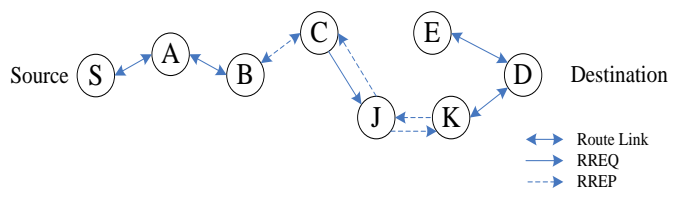

Figure 2. Example for downward route repair

\section{B. Strategy $S U$}

Fig .3 illustrates the process of upward route repair. In this scenario, the route is as follows.

$$
S \leftrightarrow A \leftrightarrow B \leftrightarrow C \leftrightarrow E \leftrightarrow D
$$

A link break occurs between nodes $\mathrm{A}$ and $\mathrm{B}$. As $\Delta H(B)=-1<1$, node $\mathrm{B}$ chooses the strategy SU according to judging rules $\mathrm{b}$ ). Within the network scale of $T T L=\operatorname{Hop}(B, S)=2$, node B broadcasts RREQ message to search for the node $\mathrm{S}$. The source IP address is set to the source node D and HC's initial value is also set to 4 . The following approach is similar to what we have mentioned above in strategy SD. With these useful control messages like RREQ and RREP, it's not difficult to resume communication. By updating the route, the new route is depicted as follows.

$$
S \leftrightarrow G \leftrightarrow F \leftrightarrow B \leftrightarrow C \leftrightarrow E \leftrightarrow D
$$

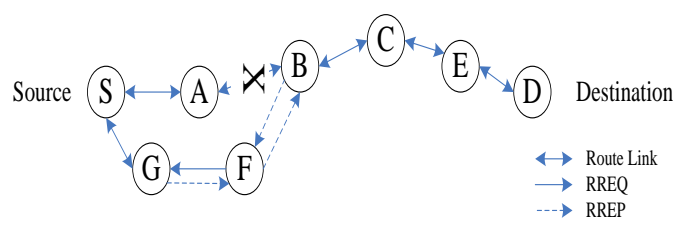

Figure 3. Example for upward route repair

\section{SimUlation AND DisCUSSION}

\section{A. Simulation Environment}

The main focus of this paper is to prove our route repair scheme's better performance. Compare AODV 
based on two-way repair ( AODV-TR) with conventional AODV protocol in Global Mobile Information System Simulator (GloMoSim2.03) tool and evaluate their performance using the metrics such as average end to end delay and average routing control cost. Table 1 shows the relevant simulation parameters.

\section{TABLE I. SIMUALTION PARAMETERS}

\begin{tabular}{c|c}
\hline Parameter & Value \\
\hline Nodes' number & 50 \\
\hline Simulation area & $1500 \mathrm{~m} \times 1000 \mathrm{~m}$ \\
\hline Mobility model & Random waypoint \\
\hline Maximum node speed & $1 \sim 60 \mathrm{~m} / \mathrm{s}$ \\
\hline Minimum node speed & 0 \\
\hline Transmission range & $250 \mathrm{metre}$ \\
\hline Physical layer & Two-way model \\
\hline MAC layer & 802.11 \\
\hline Transport layer & UDP \\
\hline Application layer & 10 flows of CBR \\
\hline Routing protocol & AODV/AODV-TR \\
\hline Data transmission rate & 5 packets/s \\
\hline Packet size & 512 byte
\end{tabular}

\section{B. Results and analysis}

Fig .4 shows an improvement of end to end delay. When the nodes were stationary, both had the same performance. As the speed increased to $30 \mathrm{~m} / \mathrm{s}$, topology changed frequently which caused the communication between nodes became more stable and longer. Both protocols' performances became better, but AODV-TR protocol had a higher level obviously because it always shortened the distance of route repair based on the route repair direction judging criterions. With the continued increasing maximum speed of nodes, the route was easy to fail due to too fast moving of nodes, causing both slowed down.

On the whole, the average delay of AODV-TR was about $99.4 \mathrm{~ms}$ and AODV was about $103.3 \mathrm{~ms}$. AODV-TR protocol outperformed AODV protocol.

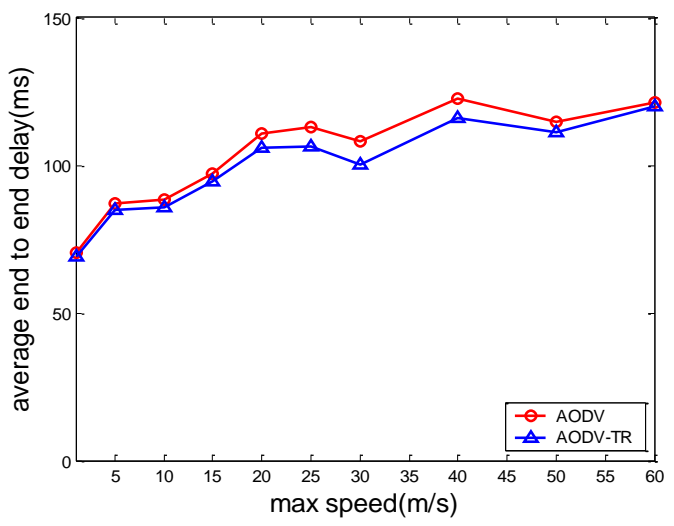

Figure 4. Comparison of average end to end delay

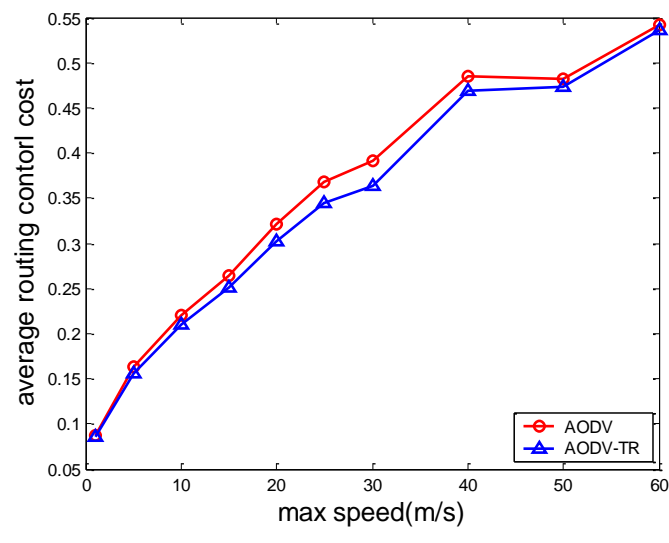

Figure 5. Comparison of routing control cost

Fig .5 shows the comparison in average routing control cost. In general, AODV-TR protocol's average control overhead accounted for about $31.9 \%$ of the total number of packets and AODV protocol was about $33.3 \%$.At the very beginning, AODV performance was close to AODV-TR. When the nodes started moving at $30 \mathrm{~m} / \mathrm{s}$, there was a shape increase in routing control cost but AODV protocol performed more poorly. At $40 \mathrm{~m} / \mathrm{s}$, communications tended to be stable and the situation got eased. However as the frequent changing positions of nodes at more than $50 \mathrm{~m} / \mathrm{s}$, both were not so well duo to the low success rate of route repair but AODV-TR protocol outperformed AODV protocol slightly.

\section{CONCLUSION}

The approach to repair the route is one of the critical factors that determine the performance of overall network in MANETs. In this paper, we propose a two-way route repair scheme. With the metrics like end to end delay and routing control cost, the simulation results show that the network performance has got improved using given route repair scheme at low node's speed, but when the nodes moved at high speed, the topology of network changes dynamically and frequent link break caused higher delay which led to both protocols performance suffering. So one of the open research questions, which we have not addressed in this paper, is how to reduce or even eliminate the effect of mobility speed on the performance of the network.

\section{REFERENCES}

[1] Perkins C, Belding-Royer E, Das S, "RFC 3561-ad hoc ondemand distance vector (AODV) routing," Internet RFCs, 2003: 138.

[2] Lee S J, Gerla M, Toh C K, "A simulation study of table-driven and on-demand routing protocols for mobile ad hoc networks," Network, IEEE, 1999, 13(4): 48-54.

[3] Lee S J, Gerla M. AODV-BR: Backup routing in ad hoc networks," Wireless Communications and Networking Confernce, 2000, 3: 1311-1316.

[4] Tambuwal A B, Noor R M, Michael O, "Improvement of DSR routing protocol using TTL-based scheme in mobile ad hoc networks," RFID-Technologies and Applications(RFID-TA), 2013: $1-6$.

[5] Rawat A, Vyavahare P D, Ramani A K, "Improved system components for secure data communication in MANETs using 
secured DSR,"Wireless Communication and Sensor Networks, 2007:61-64.

[6] Kanthe, Ashok M, Simunic, et al, "Comparsion of AODV and DSR on-demand routing protocols in mobile ad hoc networks," Emerging Technology Trends in Electronics, Communication and Networking, 2012: 1-5.

[7] Johnson D B, Maltz D A, Broch J, "DSR: The dynamic source routing protocol for multi-hop Ad hoc networking, 2001, 5: 139-172.
[8] Lu H, Guo W, "The application based on the next saved two hops in AODV routing algorithm," Computer Science, 2005, 32(4): 110-112.

[9] Xiao B L, Guo W, Liu J, et al, "Research on mobile ad hoc network based on local route repair," Journal of Computer Research and Development, 2007, 44(8): 1383-1389.

[10] Liu G, Wong K J, Lee B S, et al, "PATCH: a novel local recovery mechanism for mobile ad-hoc networks," Vehicular Technology Conference, 2003 2995-2999. 